\title{
RESPOSTAS FISIOLÓGICAS DE GENÓTIPOS DE Eucalyptus grandis x E. urophylla À DISPONIBILIDADE HÍDRICA E ADUBAÇÃ̃ POTÁSSICA
}

\author{
Hélio Sandoval Junqueira Mendes ${ }^{1}$, Nádia Figueiredo de Paula², \\ Edimar Aparecido Scarpinatti ${ }^{3}$, Rinaldo Cesar de Paula ${ }^{1 *}$
}

*Autor para correspondência: rcpaula@fcav.unesp.br

RESUMO: Conduziu-se este trabalho, com o objetivo de estudar as respostas fisiológicas de cinco genótipos de eucalipto à disponibilidade hídrica e adubação potássica. As mudas foram plantadas em vasos preenchidos com sete litros de um Neossolo Quartzarênico, com baixo teor natural de potássio $\left(0,2 \mathrm{mmol} \cdot \mathrm{dm}^{-3} \mathrm{~K}\right)$, e submetidas a dois regimes de irrigação (RI1 - diário e RI2 - irrigação suspensa até o aparecimento de sintomas iniciais de murcha), sem (K0) e com suprimento de potássio (K1 - 200 $\left.\mathrm{mg} \cdot \mathrm{dm}^{-3} \mathrm{~K}_{2} \mathrm{O}\right)$, em casa de vegetação. Avaliou-se a taxa fotossintética $(A)$, condutância estomática $(g s)$, transpiração $(E)$, índice de conteúdo de clorofila (ICC), conteúdo relativo de água (CRA), eficiência fotoquímica ( $\mathrm{Fv} / \mathrm{Fm})$, eficiência intrínseca (EUA ${ }_{\text {intr }}$ ) e instantânea no uso da água $\left(\mathrm{EUA}_{\text {inst }}\right)$ e potencial hídrico foliar $\left(\Psi_{\mathrm{f}}\right)$. O experimento foi estabelecido no delineamento de blocos ao acaso, em esquema fatorial 5 × 2 × 2 (5 genótipos, 2 regimes de irrigação e 2 níveis de adubação potássica), com cinco repetições. À exceção da eficiência fotoquímica, as demais características apresentaram alterações significativas no regime RI2, com redução nos valores de $A$, gs e $E$ e aumento em ICC e EUA inst $^{\circ} \mathrm{O}$ suprimento de potássio nas plantas do RI2 proporcionou maiores valores de $A, g s, E$ e $C R A$. Dos genótipos avaliados, o G1 é o mais resistente e o G2 o mais sensível à deficiência hídrica. Conclui-se que a adubação potássica pode amenizar os efeitos negativos da deficiência hídrica nos estádios iniciais de crescimento de eucalipto.

Palavras-chave: Fotossíntese, estresse hídrico, metabolismo fisiológico.

\section{PHYSIOLOGICAL RESPONSES OF Eucalyptus grandis $\mathrm{x}$ E. urophylla GENOTYPES TO WATER AVAILABILITY AND POTASSIUM FERTILIZATION}

\begin{abstract}
The aim of this work was to study the physiological responses of Eucalyptus genotypes seedlings to water availability and potassium fertilization. The plants were grown in pots filled with $7 \mathrm{~L}$ of the Quartzenic Neosoil, with low natural content potassium $\left(0,2 \mathrm{mmol}_{\mathrm{c}} \cdot \mathrm{dm}^{-3} \mathrm{~K}\right)$, and submitted to two irrigation regimes (IRI - diary and IR2 - cessation of watering until visible wilting occurred), no $(\mathrm{KO})$ and with $\left(\mathrm{K} 1-200 \mathrm{mg} \cdot \mathrm{dm}^{-3} \mathrm{~K}_{2} \mathrm{O}\right)$ potassium fertilization in greenhouse condition. Photosynthetic rate (A), stomatal conductance (gs), transpiration (E), chlorophyll content index (CCI), relative water content (RWC), photochemical efficiency $(F v / F m)$, intrinsic water-use-efficiency ( $i W U E)$, instantaneous water-use efficiency (WUE) and leaf water potential ( $\left.\Psi_{f}\right)$ were measured in all plants. The experiment was arranged in randomized block design, in the $5 \times 2 \times 2$ factorial schemes with five replicates. Except photochemical efficiency, all physiological traits were significantly affected by water stress with decreased in $A$, gs and $E$ values and increased in the CCI and iWUE. The application of potassium in water-stressed plants (IR2) promoted higher A, gs, E and RWC. Among genotypes examined, G1 may be considered as the most tolerant and G2 as the most susceptive to water stress. It is concluded that potassium application, could ameliorate negative effects of water stress on early growth stages of Eucalyptus genotypes.
\end{abstract}

Key words: Photosynthesis, water stress, physiological metabolism.

\section{INTRODUÇÃO}

O estresse por deficiência hídrica é um dos principais fatores que reduz o crescimento e o desenvolvimento de plantas (SEGHATOLESLAMI et al., 2008), sendo que as reações das plantas a esse fator diferem significantemente em vários níveis organizacionais, dependendo, principalmente, da intensidade e duração do estresse bem como da espécie e do estágio de crescimento da planta (CHAVES et al., 2002; JALEEL et al., 2008). Esse estresse é caracterizado pela redução do conteúdo de água, diminuição do potencial hídrico, perda do turgor, fechamento de estômatos, redução da expansão celular e do crescimento das plantas. Quando severo, o estresse por deficiência hídrica pode resultar em inibição da fotossíntese, distúrbios do metabolismo e, finalmente, na morte da planta (JALEEL et al., 2008).

Atualmente, pesquisas sobre respostas de plantas à deficiência hídrica têm se tornado cada dia mais importantes, visto que estudos de previsão de mudanças climáticas preveem a expansão de zonas áridas na terra (MISSON et al., 2010). Sendo assim, o entendimento dos

${ }^{1}$ Universidade Estadual Paulista Júlio de Mesquita Filho - Jaboticabal, São Paulo, Brasil

${ }^{2}$ Faculdade de Tecnologia - Jaboticabal, São Paulo, Brasil

${ }^{3}$ Fibria Celulose S.A. - Três Lagoas, Mato Grosso do Sul, Brasil

Cerne, Lavras, v. 19, n. 4, p. 603-611, out./dez. 2013 
efeitos da deficiência hídrica nas plantas é vital para a melhoria de práticas de manejo e melhoramento agrícola e florestal (CHAVES et al., 2003; RAMPINO et al., 2006) e uma das alternativas para amenizar seus efeitos é a seleção e uso de genótipos com características de tolerância à seca para sua introdução em programas de melhoramento genético (SILVA et al., 2007).

Dentre as práticas de manejo para obter alta produtividade nas florestas de eucalipto, uma adubação adequada é requisito essencial. O potássio, além de exercer um papel vital em um grande número de processos bioquímicos e biofísicos nas plantas, atua no controle osmótico das células. O mesmo é responsável pela mudança de turgor nas células guardas durante a abertura e fechamento dos estômatos, controlando o transporte de água com a variação da abertura estomática, afetando a difusão de água do interior das folhas para a atmosfera, bem como o fluxo contrário do $\mathrm{CO}_{2}$ na fotossíntese (MARSCHNER, 1995). No entanto, a resposta de espécies florestais à aplicação de potássio é rara, sendo que o eucalipto apresenta baixa exigência inicial nesse nutriente (BARROS et al., 1990), embora trabalhos como os de Silva et al. (2004) e Teixeira et al. (1995) evidenciam o efeito benéfico do potássio em plantas sob deficiência hídrica.

Diante do relatado, neste trabalho, objetivouse avaliar o efeito do suprimento de potássio nas características fisiológicas de genótipos de eucalipto submetidos a duas condições de disponibilidade hídrica.

\section{MATERIAL E MÉTODOS}

O experimento foi conduzido em casa de vegetação, coberta com filme de plástico transparente de 0,15 $\mu \mathrm{m}$ de espessura, revestida lateralmente com telas de sombreamento com capacidade de $30 \%$ de interceptação da luz diária, localizada na Faculdade de Ciências Agrárias e Veterinárias, da Universidade Estadual Paulista (FCAV/UNESP), Campus de Jaboticabal, no município de Jaboticabal, SP. Durante o período de condução do experimento, que teve duração de 90 dias, a temperatura média na casa de vegetação foi $18,7 \pm 8,1{ }^{\circ} \mathrm{C}$, com mínima de $12,1 \pm 3,2{ }^{\circ} \mathrm{C}$ e máxima de $28,7 \pm 2,7^{\circ} \mathrm{C}$; a umidade relativa do ar máxima variou de $96,4 \pm 2,5 \%$, a mínima de $51 \pm 24,4 \%$, com média de 79,5 $\pm 25,3 \%$.

O experimento foi estabelecido no delineamento de blocos ao acaso, em esquema fatorial $5 \times 2 \times 2$ (5 genótipos, 2 regimes de irrigação e 2 níveis de adubação potássica), com cinco repetições.

Mudas de cinco genótipos comerciais de eucalipto, doravante denominados G1, G2, G3, G4 e G5 (Tabela 1), produzidas por miniestaquia, com 80 dias, foram plantadas em vasos de plástico preenchido com sete litros de uma amostra de um Neossolo Quartzarênico, com baixo teor natural de potássio (Tabela 2). O solo, seco à sombra e peneirado, foi corrigido de forma a elevar a saturação por bases a $60 \%$, utilizando a relação $3: 1$ de $\mathrm{Ca}: \mathrm{Mg}, 20$ dias antes do plantio das mudas. Juntamente com os corretivos de acidez, aplicaram-se $200 \mathrm{mg} . \mathrm{dm}^{-3}$ de $\mathrm{P}_{2} \mathrm{O}_{5}$, na forma de superfosfato triplo, aplicados em uma única vez e incorporados manualmente ao solo. Posteriormente, o solo foi adubado, aplicando-se 30 mg.dm ${ }^{-3}$ de N; 30 mg.dm ${ }^{-3}$ de S; 5 mg.dm ${ }^{-3}$ de Zn; 0,5 mg.dm ${ }^{-3}$ de B; 0,5 mg.dm ${ }^{-3}$ de Cu; 1,5 mg.dm ${ }^{-3}$ de Mn; e $0,1 \mathrm{mg} \cdot \mathrm{dm}^{-3}$ de Mo. Metade dos vasos não recebeu complementação potássica (K0) e a outra metade recebeu $200 \mathrm{mg} \cdot \mathrm{dm}^{-3} \mathrm{~K}_{2} \mathrm{O}$ (K1) via cloreto de potássio, em solução, em uma única dose.

Tabela 1 - Relação de genótipos avaliados.

Tabela 1 - Relation of the evaluated genotypes.

\begin{tabular}{lcccc}
\hline \multirow{2}{*}{ Genótipos } & \multicolumn{2}{c}{ Genitor Feminino } & \multicolumn{2}{c}{ Genitor Masculino } \\
\cline { 2 - 4 } & Origem & Procedência $^{2}$ & Origem $^{1}$ & Procedência $^{2}$ \\
\hline G1 - E. grandis $x$ E. urophylla & Coffs Harbour & Santa Bárbara & Timor & Luiz Antônio \\
G2 - E. grandis $x$ E. urophylla & Coffs Harbour & Altinópolis & Timor & Luiz Antônio \\
G3 - E. grandis $x$ E. urophylla & Coffs Harbour & Altinópolis & Timor & São Simão \\
G4 - E. urophylla $x$ E. grandis & Flores & Mogi-Guaçu & Coffs Harbour & Mogi-Guaçu \\
G5 - E. urophylla $x$ E. grandis & Flores & Mogi-Guaçu & Coffs Harbour & Mogi-Guaçu \\
\hline
\end{tabular}

1 - Origem: Coffs Harbour - Austrália; Flores e Timor - Indonésia

${ }^{2}$ - Todas as procedências referem-se a municípios do Estado de São Paulo

Cerne, Lavras, v. 19, n. 4, p. 603-611, out./dez. 2013 
Tabela 2 - Análise de uma amostra do Neossolo Quartzarênico para fins de fertilidade e granulometria.

Table 2 - Analyses for particle size and fertility of the sample of soil.

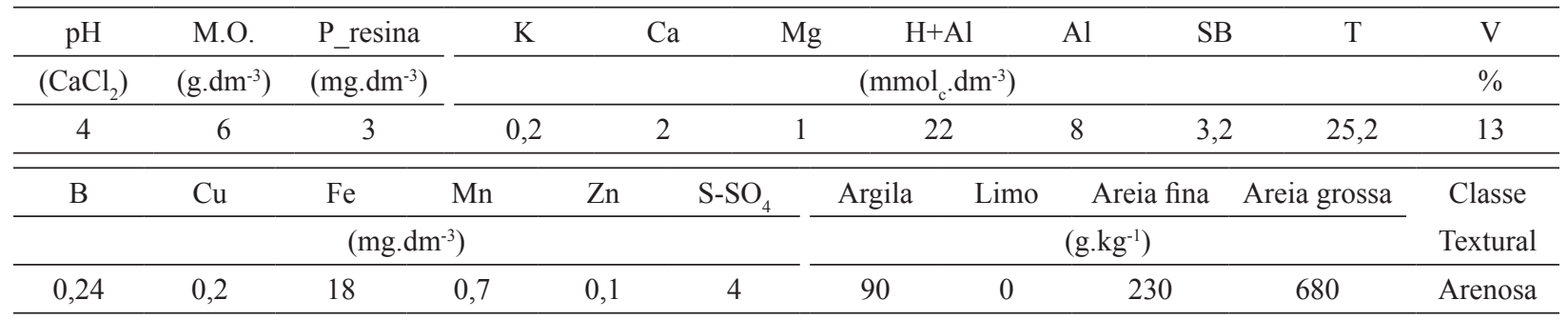

Após o plantio, as mudas passaram por um período de aclimatação de seis dias, sendo, então, estabelecidos os dois regimes de irrigação: RI1 - irrigação diária de forma a manter $60 \%$ da capacidade máxima de retenção de água pelo solo, ou seja, plantas sem restrição hídrica e RI2 - plantas irrigadas até $60 \%$ da capacidade de retenção de água do solo, com posterior suspensão da irrigação até o aparecimento de sintomas iniciais de deficiência hídrica (desidratação/murcha; curvatura da gema terminal). As plantas do regime hídrico RI2, ao final do ciclo de suspensão da irrigação, foram irrigadas até o solo atingir $60 \%$ da capacidade de retenção de água, suspendendo, novamente, a irrigação, repetindo-se o ciclo. No decorrer do experimento, foram conduzidos sete ciclos de suspensão de irrigação, sendo a quantidade de água reposta em cada vaso determinada pelo método gravimétrico.

Ao final de cada ciclo de suspensão de irrigação, foram avaliadas em todas as plantas do experimento as seguintes características: índice de conteúdo de clorofila (ICC), usando um clorofilômetro portátil (CCM 200, Opti Science); eficiência fotoquímica do fotossistema II, dada pela razão $\mathrm{Fv} / \mathrm{Fm}$, usando um fluorômetro portátil (OS30p, Opti Science), taxa fotossintética $(A)$, condutância estomática $(g S)$ e transpiração $(E)$. Para a avaliação de $A$, gs e $E$ foi usado um analisador de gases a infravermelho, portátil (LCPro ${ }^{+}$SYSTEM, ADC) sob concentração de $\mathrm{CO}_{2}$ ambiente $\left(370 \pm 10 \mu \mathrm{mol} . \mathrm{m}^{-2} \mathrm{~s}^{-1}\right)$, FFFA (fluxo de fótons fotossinteticamente ativo) de $1000 \mu \mathrm{mol} . \mathrm{m}^{-2} \cdot \mathrm{s}^{-1} \mathrm{e}$ temperatura da folha mantida a $26^{\circ} \mathrm{C}$. As medições foram realizadas nos períodos compreendidos entre $8 \mathrm{~h}$ e $10 \mathrm{~h}$ e 30 min, em folhas totalmente expandidas, no terço médio das plantas. A partir das avaliações de $A, g s$ e $E$, foram obtidas as eficiências instantânea $\left(\mathrm{EUA}_{\text {inst }}=A / g s\right)$ e a eficiência intrínseca $\left(\mathrm{EUA}_{\text {intr }}=A / E\right)$ do uso da água, conforme Ngugi et al. (2003a, 2003b, 2004). Também, determinou-se o conteúdo relativo de água (CRA) de acordo com Catsky
(1974), em seis ciclos de suspensão da irrigação, e o potencial hídrico foliar $\left(\Psi_{\mathrm{f}}\right)$ ao final do experimento, por meio de uma bomba de pressão (SCHOLANDER et al., 1965).

As análises de variância foram realizadas considerando-se as médias dos ciclos de suspensão de irrigação, usando-se o programa SISVAR (FERREIRA, 2000) e as médias de tratamentos foram comparadas pelo teste de Tukey a $5 \%$ de probabilidade.

\section{RESULTADOS E DISCUSSÃO}

Houve modificações $(P \leq 0,01 ; P \leq 0,05)$ nas características avaliadas quando a irrigação foi suspensa até o aparecimento de sintomas iniciais de deficiência hídrica (regime de irrigação 2 - RI2), tanto de forma isolada, como observado para eficiência intrínseca no uso da água $\left(\mathrm{EUA}_{\text {intr }}\right)$ e potencial hídrico foliar $\left(\Psi_{\mathrm{f}}\right)$, como em interações com genótipos e/ou com adubação potássica para as demais características (Tabela 3). Na média dos cinco genótipos e dos dois níveis de adubação potássica, houve reduções de $259 \%$ no $\Psi_{\mathrm{f}}$ e aumento de $6,5 \%$ na EUA $_{\text {intr }}$ no RI2.

$\mathrm{O}$ efeito da adubação potássica foi pequeno, mas significativo $(P \leq 0,01 ; P \leq 0,05)$ para taxa fotossintética $(A)$, transpiração $(E)$, conteúdo relativo de água (CRA), índice de conteúdo de clorofila (ICC), eficiência fotoquímica $(\mathrm{Fv} / \mathrm{Fm})$ e $\mathrm{EUA}_{\text {intr }}$. Dessas características, houve efeito isolado da adubação apenas sobre EUA $_{\text {intr }}$, com maiores valores observados na presença de K (K1). Também foi observada interação RI x K significativa $(P \leq 0,01)$ sobre a condutância estomática $(g s)$.

Na média dos dois regimes de irrigação e dos dois níveis de adubação potássica, os genótipos diferiram $(P \leq 0,01 ; P \leq 0,05)$ na maioria das características. Para condutância estomática, o G1 apresentou maiores médias que os demais genótipos, que não diferiram entre si.

Cerne, Lavras, v. 19, n. 4, p. 603-611, out./dez. 2013 
Tabela 3 - Valores de "F" da análise de variância, coeficientes de variação (CV, \%) e médias das características avaliadas em cinco genótipos de eucalipto $(\mathrm{G})$ submetidos a dois regimes de irrigação (RI) e na ausência (K0) e presença (K1) de adubação potássica (K).

Table 3 - Values of " $F$ " of the variance analysis, variation coefficients (CV, \%) and means of the traits evaluated in five eucalypt genotypes $(G)$ growing in two irrigations regimes $(R I)$ in the absence (K0) and presence (K1) of potassium fertilization (K).

\begin{tabular}{|c|c|c|c|c|c|c|c|c|c|}
\hline \multirow{2}{*}{$\begin{array}{l}\text { Fontes de } \\
\text { Variação }\end{array}$} & \multicolumn{9}{|c|}{ Variáveis } \\
\hline & $A$ & $g s$ & $E$ & EUA $_{\text {inst }}$ & EUA $_{\text {intr }}$ & $\Psi_{\mathrm{f}}$ & CRA & ICC & $\mathrm{Fv} / \mathrm{Fm}$ \\
\hline RI & $899,9 * *$ & $1940,7^{* *}$ & $1583,1^{* *}$ & $1431,3 * *$ & $15,3 * *$ & $992,9 * *$ & $132,3 * *$ & $677,2 * *$ & $139,4 * *$ \\
\hline K & $20,1 * *$ & $0,1^{\mathrm{ns}}$ & $6,0 * *$ & $0,1^{\mathrm{ns}}$ & $7,6^{* *}$ & $0,1^{\mathrm{ns}}$ & $7,2 * *$ & $16,3^{* *}$ & $6,1^{*}$ \\
\hline $\mathrm{G}$ & $14,7 * *$ & $14,9 * *$ & $12,6^{* *}$ & $5,7 * *$ & $1,4^{\mathrm{ns}}$ & $0,5^{\mathrm{ns}}$ & $7,8 * *$ & $40,8 * *$ & $54,6^{* *}$ \\
\hline RI x K & $7,1 * *$ & $7,4^{* *}$ & $16,7 * *$ & $0,9^{\mathrm{ns}}$ & $0,2^{\text {ns }}$ & $0,4^{\mathrm{ns}}$ & $7,2 * *$ & $6,8^{*}$ & $0,1^{\mathrm{ns}}$ \\
\hline RI x G & $4,3 * *$ & $0,9^{\mathrm{ns}}$ & $2,9^{*}$ & $2,7^{*}$ & $0,6^{\mathrm{ns}}$ & $0,9^{\text {ns }}$ & $7,4 * *$ & $10,5^{* *}$ & $7,7 * *$ \\
\hline $\mathrm{K} \times \mathrm{G}$ & $0,8^{\mathrm{ns}}$ & $0,5^{\mathrm{ns}}$ & $0,9^{\text {ns }}$ & $1,6^{\mathrm{ns}}$ & $1,6^{\mathrm{ns}}$ & $0,2^{\mathrm{ns}}$ & $1,2^{\mathrm{ns}}$ & $0,9^{\text {ns }}$ & $1,4^{\mathrm{ns}}$ \\
\hline $\mathrm{RI} \times \mathrm{K} \times \mathrm{G}$ & $0,6^{\mathrm{ns}}$ & $1,3^{\mathrm{ns}}$ & $1,4^{\mathrm{ns}}$ & $1,9^{\text {ns }}$ & $0,7^{\mathrm{ns}}$ & $0,4^{\mathrm{ns}}$ & $0,4^{\mathrm{ns}}$ & $1,8^{\mathrm{ns}}$ & $3,2 *$ \\
\hline $\mathrm{CV}(\%)$ & 9,83 & 14,44 & 8,71 & 13,49 & 8,09 & 18,00 & 3,12 & 9,47 & 0,50 \\
\hline Média & 11,76 & 0,394 & 2,76 & 50,14 & 3,47 & $-1,24$ & 86,4 & 27,1 & 0,836 \\
\hline \multicolumn{10}{|c|}{ 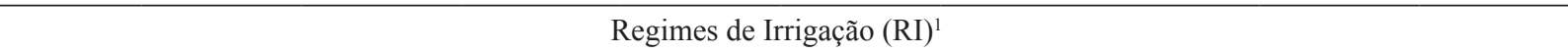 } \\
\hline RI1 & 15,2 & 0,64 & 3,71 & 24,5 & $3,36 \mathrm{~B}$ & $-0,54 \mathrm{~B}$ & 89,4 & 20,4 & 0,830 \\
\hline $\mathrm{RI} 2$ & 8,3 & 0,14 & 1,80 & 75,7 & $3,58 \mathrm{~A}$ & $-1,94 \mathrm{~A}$ & 83,2 & 33,7 & 0,840 \\
\hline \multicolumn{10}{|c|}{ Níveis de potássio (K) } \\
\hline K0 & 11,2 & 0,39 & 2,70 & 50,0 & $3,39 \mathrm{~B}$ & $-1,24$ & 85,6 & 28,1 & 0,834 \\
\hline K1 & 12,3 & 0,39 & 2,81 & 50,2 & $3,55 \mathrm{~A}$ & $-1,25$ & 87,0 & 26,0 & 0,836 \\
\hline \multicolumn{10}{|c|}{ Genótipos $(\mathrm{G})$} \\
\hline G1 & 13,1 & $0,47 \mathrm{~A}$ & 3,04 & 44,2 & 3,49 & $-1,25$ & 87,3 & 23,0 & 0,840 \\
\hline G2 & 10,5 & $0,34 \mathrm{~B}$ & 2,55 & 50,0 & 3,35 & $-1,27$ & 84,2 & 31,7 & 0,823 \\
\hline G3 & 11,2 & $0,36 \mathrm{~B}$ & 2,63 & 53,8 & 3,45 & $-1,19$ & 84,8 & 29,0 & 0,837 \\
\hline G4 & 11,9 & $0,39 \mathrm{~B}$ & 2,74 & 51,9 & 3,52 & $-1,22$ & 87,6 & 27,7 & 0,839 \\
\hline G5 & 12,0 & $0,38 \mathrm{~B}$ & 2,82 & 50,7 & 3,55 & $-1,27$ & 87,7 & 23,8 & 0,838 \\
\hline
\end{tabular}

RI1 - irrigação diária; RI2 - irrigação suspensa até o aparecimento de sintomas iniciais de deficiência hídrica; K0 - sem adição de $\mathrm{K}_{2} \mathrm{O} ; \mathrm{K} 1$ - adição de $200 \mathrm{mg} \cdot \mathrm{dm}^{-3} \mathrm{~K}_{2} \mathrm{O} ; A$ - taxa fotossintética $\left(\mu \mathrm{mol} \cdot \mathrm{m}^{-2} \cdot \mathrm{s}^{-1}\right) ; g s$ - condutância estomática $\left(\mathrm{mol} \cdot \mathrm{m}^{-2} \cdot \mathrm{s}^{-1}\right) ; E$ - transpiração $\left(\mathrm{mmol} \cdot \mathrm{m}^{-2} \cdot \mathrm{s}^{-1}\right)$ EUA $_{\text {inst }}$-eficiência instantânea $\left(\mu \mathrm{mol} \cdot \mathrm{mol}^{-1}\right)$ no uso da água; EUA $\mathrm{intr}_{\text {- }}$ eficiência intrínseca no uso da água ( $\mu$ mol. $\left.\mathrm{mmol}^{-1}\right) ; \Psi_{\mathrm{f}}$ - potencial hídrico foliar (MPa); CRA - conteúdo relativo de água nas folhas (\%); ICC - índice de conteúdo de clorofila; $\mathrm{Fv} / \mathrm{Fm}$ - eficiência fotoquímica do fotossistema II (Fv/Fm).

$*-p \leq 0,05 ; * *-p \leq 0,01$ e ns $-\mathrm{p}>0,05$, respectivamente pelo teste $\mathrm{F}$.

${ }^{1}$ - médias seguidas por uma mesma letra, em cada fator principal, não diferem pelo teste de Tukey a 5\%.

No regime hídrico 1 (RI1), a taxa fotossintética $(A)$, condutância estomática $(g s)$, transpiração $(E)$, conteúdo relativo de água (CRA) e índice de conteúdo de clorofila (ICC) não diferiram entre os dois níveis de adubação potássica; por outro lado no RI2, as plantas que receberam adubação apresentaram maiores valores nessas características (Tabela 4). Também, observa-se que, independentemente da adubação, o RI2 proporcionou reduções significativas nessas características, exceto no
ICC. Dessa forma, pode-se inferir que, logo após o plantio, no período de estabelecimento da cultura do eucalipto, desde que não ocorra deficiência hídrica, a resposta à adubação potássica é pouco provável.

O efeito da adubação potássica, tanto sobre o crescimento quanto sobre as características fisiológicas, pode variar em função de uma série de fatores a exemplo da espécie (TEIXEIRA et al., 2006, 2008), da textura e do teor de potássio do solo (BARROS et al., 1990; NEVES

Cerne, Lavras, v. 19, n. 4, p. 603-611, out./dez. 2013 
Tabela 4 - Médias de taxa fotossintética $\left(A\right.$, em $\mu$ mol.m $\left.\mathrm{m}^{-2} \cdot \mathrm{s}^{-1}\right)$, condutância estomática $\left(g s\right.$, em mol.m $\left.{ }^{-2} \cdot \mathrm{s}^{-1}\right)$, transpiração $(E$, em mmol. $\left.\mathrm{m}^{-2} \cdot \mathrm{s}^{-1}\right)$, conteúdo relativo de água (CRA, \%) e índice de conteúdo de clorofila (ICC) em cinco genótipos de eucalipto submetidos a dois regimes de irrigação (RI1 e RI2) na ausência (K0) e presença (K1) de adubação potássica.

Table 4 - Means of photosynthetic rate $\left(A, \mu \mathrm{mol} . \mathrm{m}^{-2} . \mathrm{s}^{-1}\right)$, stomata conductance $\left(\mathrm{gs}, \mathrm{mol} . \mathrm{m}^{-2} \cdot \mathrm{s}^{-1}\right)$, transpiration rate $\left(E, \mathrm{mmol}^{-m^{-2}} . \mathrm{s}^{-1}\right)$, relative water content (CRA, \%) and chlorophyll content index (ICC) evaluated in five eucalypt genotypes growing in two irrigations regimes (RI1 e RI2) in the absence (KO) and presence (K1) of the potassium fertilization $(K)$.

\begin{tabular}{|c|c|c|c|c|c|c|c|c|c|c|}
\hline & \multicolumn{2}{|c|}{$A$} & \multicolumn{2}{|c|}{$g s$} & \multicolumn{2}{|c|}{$E$} & \multicolumn{2}{|c|}{ CRA } & \multicolumn{2}{|c|}{ ICC } \\
\hline & K0 & K1 & K0 & $\mathrm{K} 1$ & K0 & $\mathrm{K} 1$ & K0 & $\mathrm{K} 1$ & K0 & K1 \\
\hline RI1 & $15,0 \mathrm{Aa}$ & $15,4 \mathrm{Aa}$ & $0,65 \mathrm{Aa}$ & $0,62 \mathrm{Aa}$ & $3,75 \mathrm{Aa}$ & $3,67 \mathrm{Aa}$ & $89,4 \mathrm{Aa}$ & $89,4 \mathrm{Aa}$ & $20,7 \mathrm{Ab}$ & $20,0 \mathrm{Ab}$ \\
\hline RI2 & $7,46 \mathrm{Bb}$ & $9,12 \mathrm{Ab}$ & $0,12 \mathrm{Bb}$ & $0,15 \mathrm{Ab}$ & $1,64 \mathrm{Bb}$ & $1,96 \mathrm{Ab}$ & $81,8 \mathrm{Bb}$ & $84,7 \mathrm{Ab}$ & $35,4 \mathrm{Aa}$ & $32,0 \mathrm{Ba}$ \\
\hline
\end{tabular}

Letras maiúsculas comparam níveis de potássio dentro de regimes de irrigação e letras minúsculas comparam regimes de irrigação dentro de cada nível de potássio (RI1 - irrigação diária; RI2 - irrigação suspensa até o aparecimento de sintomas iniciais de deficiência hídrica; K0 - sem adição de $\mathrm{K}_{2} \mathrm{O} ; \mathrm{K} 1$ - adição de $200 \mathrm{mg} \cdot \mathrm{dm}^{-3} \mathrm{~K}_{2} \mathrm{O}$ ).

et al., 1990; NOVAIS et al., 1990), do volume de solo disponível para o crescimento das plantas (NEVES et al., 1990; NOVAIS et al., 1990) e da própria umidade do solo. Assim, em pequenos volumes de solos, a exemplo de tubetes, sacos de plástico e mesmo em vasos de pequenas dimensões, a probabilidade de se observar efeito da adubação com $\mathrm{K}$ é maior do que em volumes maiores, em função não do teor, mas da quantidade disponível de $\mathrm{K}$ para as plantas.

Romero (2008), trabalhando com mudas de Eucalyptus grandis submetidas a duas tensões de água, em vasos preenchidos com $5 \mathrm{Kg}$ de uma amostra de um Latossolo Vermelho-Amarelo distrófico típico, textura média, e combinando a adubação com potássio e sódio, observou que independentemente da disponibilidade de água no solo, a adubação potássica promoveu maior crescimento em altura e em diâmetro nas plantas, mas que esse efeito foi potencializado sob condições adequadas de água disponível. Teixeira et al. (2006), trabalhando com mudas de E. grandis, E. urophylla, E. camaldulensis e de um híbrido entre E. urophylla $x E$. grandis, submetidas a quatro doses de $\mathrm{K}(0,50,100 \mathrm{e}$ $200 \mathrm{mg} . \mathrm{dm}^{-3}$ de K) e dois regimes de umidade do solo (-0,01MPa: "sem estresse hídrico" e -0,10 MPa: "com estresse hídrico"), em vasos contendo $3 \mathrm{dm}^{3}$ de uma amostra de um Latossolo Vermelho-Amarelo distrófico típico, textura média, encontraram que o estresse hídrico não afetou significativamente o crescimento em altura de E. urophylla, E. grandis e do híbrido E. urophylla $\mathrm{x} E$. grandis, mas reduziu a matéria seca da parte aérea de todas as espécies. Por outro lado, a adição de potássio ao solo afetou fortemente o crescimento em altura e a produção de matéria seca das espécies estudadas, tanto com e sem estresse hídrico.
Sob deficiência hídrica, as plantas que receberam adubação potássica (K1), apresentam melhor desempenho fisiológico que aquelas não adubadas, apresentando melhor controle estomático e, consequentemente, maiores valores de $A$ e $E$ (Tabela 4). Segundo Marschner (1995), o decréscimo da fotossíntese em condições de estresse hídrico é menor quando as plantas estão bem supridas de potássio, pois o aumento do conteúdo de potássio nas folhas proporciona maior atividade da ribulose bifosfato carboxilase/oxigenase (rubisco) e maior taxa fotossintética. Fanaei et al. (2009) em estudo com Brassica napus e $B$. juncea, submetidas a três níveis de irrigação e a três níveis de adubação potássica, observaram que a aplicação de K pode minimizar os efeitos negativos da deficiência hídrica sobre a produção de grãos e aspectos fisiológicos das plantas. Teixeira et al. (1995), estudando diversas espécies e híbridos de Eucalyptus, verificaram que sob estresse hídrico e fornecimento de potássio, a média do CRA nas folhas das espécies de Eucalyptus foi de 81,7\% contra somente 67,3\% na ausência do elemento. De forma isolada, o déficit hídrico diminuiu o CRA de seis espécies de eucalipto, porém de forma diferenciada entre elas (MERCHANT et al., 2007). Também, Teixeira et al. (2008) trabalhando com mudas de E. grandis, E. urophylla, E. camaldulensis e de um híbrido entre $E$. urophylla $x$ E. grandis, encontraram que a aplicação de $\mathrm{K}$ influenciou a taxa fotossintética $(A)$, a condutância estomática $(g s)$, a transpiração $(E)$ e a eficiência no uso da água (EUA). De acordo com esses autores, plantas deficientes em K apresentaram menor $A$ e maior $g s$ e $E$, com consequente menor EUA; não houve diferenças estatísticas na $A, g s$ e $E$ entre plantas com e sem déficit hídrico, mas a adição de $\mathrm{K}$ diminuiu o consumo de água por unidade de área foliar e, em geral, plantas submetidas à deficiência hídrica apresentaram menor consumo de água.

Cerne, Lavras, v. 19, n. 4, p. 603-611, out./dez. 2013 
Sob irrigação diária, os genótipos G1 e G4 apresentaram maior valor de $A$ que o genótipo G2 e para $E$ não houve diferenças entre os genótipos; no RI2, o genótipo G1 superou os demais genótipos e o G2 apresentou o menor valor de $A$ e $E$, diferindo do G5, mas não do G3 e G4. Todos os genótipos apresentaram reduções significativas em $A$ no RI2 (Tabela 5). A EUA inst foi maior no RI2, independentemente do genótipo; neste regime de irrigação, o genótipo G1 apresentou o menor valor de EUA $_{\text {inst }}$ No RI1, não houve diferença entre os genótipos. Reduções semelhantes em $A, g s, E$ e $\Psi_{\mathrm{f}}$ foram observadas em clones de eucalipto sob diferentes disponibilidades hídricas (TATAGIBA et al., 2008).

Estudando seis espécies de eucalipto, Merchant et al. (2007) observaram reduções mais acentuadas na condutância estomática do que na taxa fotossintética. Em E. tricarpa e E. camaldulensis, foram observados maiores valores de potencial hídrico e de conteúdo relativo de água nas folhas, manifestando menos os efeitos da deficiência hídrica. Houve redução no acúmulo de matéria seca nas seis espécies e ajuste da área transpiracional de acordo com a disponibilidade hídrica. Para esses autores, redução simultânea do potencial osmótico (redistribuição dos constituintes celulares) e da área foliar total sugere uma coordenação de adaptações fisiológicas e morfológicas. Possivelmente, essas características poderão conferir alguma vantagem a essas espécies quando plantadas em condições de seca.

Maiores valores de ICC ocorreram no RI2 e, nesse regime, o genótipo G2 superou os demais e G1 e G5 apresentaram os menores valores de ICC. No RI1 o G2 superou os genótipos G1 e G5, não havendo diferenças entre os demais genótipos. $\mathrm{O}$ aumento do ICC nas plantas do RI2 (Tabela 5) é, provavelmente, decorrente do menor crescimento observado nessa condição e, portanto, ao maior teor de $\mathrm{N}$ nas folhas, o que corrobora com Silva et al. (2004), que encontraram altos teores de clorofila em mudas de Eucalyptus grandis submetidos a estresse hídrico mais severo.

No RI2, houve inibição da fotossíntese $(A)$, como consequência do fechamento dos estômatos, evidenciado pelos menores valores de gs (Tabelas 4 e 5). O estresse hídrico afeta a fotossíntese, tanto pelo fechamento dos estômatos, limitando a troca gasosa, como pelo comprometimento metabólico (FLEXAS et al., 2002). A forte relação entre gs e vários parâmetros fotossintéticos levaram Flexas e Medrano (2002) a sugerirem o uso de condutância estomática como um parâmetro de referência para avaliar o grau de estresse hídrico. Além disso, a condutância estomática pode ser uma ferramenta útil no melhoramento genético para maior produtividade em ambientes propensos à seca, por fornecer uma ligação crucial entre a perda de água pela transpiração e ganho de carbono (e, portanto, do crescimento) e entre a economia de água pelo fechamento dos estômatos e maior estresse por calor (PITA et al., 2005).

As características mais sensíveis à suspensão da irrigação foram o $\Psi_{\mathrm{f}}$, seguido de $g s, E$ e $A$. O $\Psi_{\mathrm{f}}$ indica o grau de hidratação de uma planta e reflete as condições da dinâmica do processo do transporte no sistema solo-águaplanta, constituindo o principal componente responsável pelo fluxo de água, podendo representar o estresse hídrico que a planta está submetida (PEREIRA et al., 2006).

Tabela 5 - Médias de taxa fotossintética $\left(A\right.$, em $\left.\mu \mathrm{mol} \cdot \mathrm{m}^{-2} \cdot \mathrm{s}^{-1}\right)$, transpiração $\left(E\right.$, em mmol. $\left.\mathrm{m}^{-2} \cdot \mathrm{s}^{-1}\right)$, conteúdo relativo de água $(\mathrm{CRA}, \%)$, eficiência instantânea no uso da água $\left(\mathrm{EUA}-\mathrm{inst}=A / g s\right.$, em $\left.\mu \mathrm{mol} \cdot \mathrm{mol}^{-1}\right)$, índice de conteúdo de clorofila (ICC) e eficiência fotoquímica (Fv/Fm) avaliados em cinco genótipos de eucalipto (G1,.., G5) em dois regimes de irrigação (RI1; RI2).

Table 5 - Means of photosynthetic rate $\left(A, \mu\right.$ mol. $\left.m^{-2} \cdot s^{-1}\right)$, transpiration rate $\left(E\right.$, mmol. $\left.m^{-2} \cdot s^{-1}\right)$, instantaneous water use efficiency $\left(E U A_{\text {inst }}=A / g s, \mu m o l . m o l^{-1}\right)$ relative water content $(C R A, \%)$, chlorophyll content index (ICC) and photochemical efficiency $(F v / F m)$ evaluated in five eucalypt genotypes $(G 1, \ldots, G 5)$ in two irrigations regimes (RI1 e RI2).

\begin{tabular}{|c|c|c|c|c|c|c|c|c|c|c|c|c|}
\hline & \multicolumn{2}{|c|}{$A$} & \multicolumn{2}{|c|}{$E$} & \multicolumn{2}{|c|}{ EUA $_{\text {inst }}$} & \multicolumn{2}{|c|}{ CRA } & \multicolumn{2}{|c|}{ ICC } & \multicolumn{2}{|c|}{$\mathrm{Fv} / \mathrm{Fm}$} \\
\hline & RI1 & RI2 & RI1 & RI2 & RH1 & RI2 & RI1 & RI2 & RI1 & RH2 & RI1 & RI2 \\
\hline G1 & $15,7 \mathrm{Aa}$ & $10,5 \mathrm{Ba}$ & $3,85 \mathrm{Aa}$ & $2,23 \mathrm{Ba}$ & $22,0 \mathrm{Ba}$ & $66,3 \mathrm{Ab}$ & $89,0 \mathrm{Aa}$ & $85,6 \mathrm{Ba}$ & $17,9 \mathrm{Bb}$ & $28,0 \mathrm{Ac}$ & $0,836 \mathrm{Ba}$ & $0,843 \mathrm{Aab}$ \\
\hline G2 & $14,0 \mathrm{Ab}$ & $6,9 \mathrm{Bc}$ & 3,59 Aa & $1,50 \mathrm{Bc}$ & $24,9 \mathrm{Ba}$ & $75,1 \mathrm{Aa}$ & $89,6 \mathrm{Aa}$ & $78,9 \mathrm{Bb}$ & $22,9 \mathrm{Ba}$ & $40,6 \mathrm{Aa}$ & $0,816 \mathrm{Bc}$ & $0,830 \mathrm{Ac}$ \\
\hline G3 & 15,1 Aab & 7,3 Bbc & 3,63 Aa & $1,62 \mathrm{Bbc}$ & $25,3 \mathrm{Ba}$ & $82,3 \mathrm{Aa}$ & $89,2 \mathrm{Aa}$ & $80,5 \mathrm{Bb}$ & $21,0 \mathrm{Bab}$ & $37,1 \mathrm{Ab}$ & $0,830 \mathrm{Bb}$ & $0,844 \mathrm{Aab}$ \\
\hline G4 & $15,8 \mathrm{Aa}$ & $8,0 \mathrm{Bbc}$ & $3,75 \mathrm{Aa}$ & $1,74 \mathrm{Bbc}$ & $24,5 \mathrm{Ba}$ & $79,3 \mathrm{Aa}$ & $89,4 \mathrm{Aa}$ & $85,9 \mathrm{Ba}$ & $20,9 \mathrm{Bab}$ & $34,7 \mathrm{Ab}$ & 0,833 Bab & $0,846 \mathrm{Aa}$ \\
\hline G5 & $15,4 \mathrm{Aab}$ & $8,7 \mathrm{Bb}$ & 3,73 Aa & $1,90 \mathrm{Bb}$ & $25,9 \mathrm{Ba}$ & $75,6 \mathrm{Aa}$ & $90,1 \mathrm{Aa}$ & $85,3 \mathrm{Ba}$ & $19,3 \mathrm{Bb}$ & $28,3 \mathrm{Ac}$ & $0,837 \mathrm{Ba}$ & $0,839 \mathrm{Ab}$ \\
\hline
\end{tabular}

Letras maiúsculas comparam regimes de irrigação (RI) dentro de cada genótipo e letras minúsculas comparam genótipos dentro de cada regime de irrigação. . RI1 - irrigação diária; RI2 - irrigação suspensa até o aparecimento de sintomas iniciais de deficiência hídrica.

Cerne, Lavras, v. 19, n. 4, p. 603-611, out./dez. 2013 
Segundo Mielke et al. (1999), é provável que a condutância estomática dependa do potencial hídrico da folha, que diminui sob condições de estresse. A diminuição da $g s$ e $E$ e, por fim, da taxa fotossintética em consequência do fechamento estomático são as primeiras respostas para reduzir a perda da água pelas plantas sob deficiência hídrica (CHAVES et al., 2003; PITA et al., 2005). Por outro lado, o aumento na EUA $\mathrm{inst}_{\text {int }}$ com a intensificação da deficiência hídrica deve-se mais à redução proporcionalmente maior em $g s$ do que a observada em $A$. O mesmo raciocínio é válido para a $\mathrm{EUA}_{\text {intr }}$ em que, de acordo com Pita et al. (2005), a maior eficiência do uso da água pode estar relacionada ou a um maior crescimento e/ou fotossíntese ou a maior taxa de fechamento dos estômatos. Ainda, para esses autores, a tendência da EUA ${ }_{\text {intr }}$ de aumentar com os estômatos fechados, significa que selecionar para aumento da EUA $_{\text {intr }}$ pode, muitas vezes, ser o mesmo que selecionar para baixa produtividade. Em oliveiras (Olea europaea L.) foi observado aumento na eficiência no uso da água com a aplicação de $\mathrm{K}$, tanto em plantas irrigadas quanto sob deficiência hídrica, porém, de forma mais expressiva nas últimas (ARQUERO et al., 2006).

O conteúdo relativo de água não diferiu entre os genótipos no RI1, mas no RI2 os genótipos G2 e G3 apresentaram valores inferiores de CRA, podendo-se supor que esses genótipos detêm menor capacidade de economizar água. Merchant et al. (2007) observaram reduções expressivas no CRA de seis espécies de eucalipto sob quatro regimes de irrigação.

As diferenças estatísticas observadas para a eficiência fotoquímica $(\mathrm{Fv} / \mathrm{Fm})$, deve-se à baixa variabilidade dos dados e ao baixo coeficiente de variação (Tabelas 3 e 6) e não ao efeito dos tratamentos, de forma que pode se afirmar não ter ocorrido danos ao fotossistema II, pela deficiência hídrica ou à falta de suprimento de $\mathrm{K}$. Segundo Rascher et al. (2000), a eficiência fotoquímica depende essencialmente da intensidade de luz, desta forma, uma possível explicação para esse resultado é que o experimento foi conduzido em casa de vegetação, amenizando, assim, a radiação que incide sobre as plantas. Resultados semelhantes foram obtidos por Rolando e Little (2008) que observaram que o estresse hídrico não afetou a relação Fv/Fm em plantas de Eucalyptus grandis, contrariando resultados anteriores obtidos pelos mesmos autores e com a mesma espécie (ROLANDO; LITTLE, 2003). Para esses autores, a falta de alterações detectáveis na eficiência fotoquímica pode estar associada a maior tolerância ao estresse hídrico das plantas usadas nesse último experimento, ou a condução do experimento por um período insuficiente para induzir alterações que pudessem ser detectadas. Em mudas de Picea abies, redução significativa de $\mathrm{Fv} / \mathrm{Fm}$ foi observada, somente, durante as fases mais avançadas do estresse hídrico imposto (DITMAROVÁ et al., 2010).

Tabela 6-Médias da eficiência fotoquímica (Fv/Fm) avaliada em cinco genótipos de eucalipto (G1, G2, G3, G4 e G5) submetidos a dois regimes de irrigação (RI1 e RI2) na ausência (K0) e presença (K1) de adubação potássica.

Table 6-Means of photochemical efficiency $(F v / F m)$ evaluated in five eucalypt genotypes (G1, G2, G3, G4 and G5) growing in two irrigations regimes (RII and RI2) in the absence (KO) and presence (K1) of the potassium fertilization (K).

\begin{tabular}{|c|c|c|c|c|}
\hline & \multicolumn{2}{|c|}{ RI1 } & \multicolumn{2}{|c|}{ RI2 } \\
\hline & K0 & K1 & K0 & K1 \\
\hline G1 & $0,838 \mathrm{Aa}$ & $0,835 \mathrm{Aa}$ & $0,841 \mathrm{Aab}$ & $0,845 \mathrm{Aa}$ \\
\hline G2 & $0,817 \mathrm{Ac}$ & $0,815 \mathrm{Aa}$ & $0,829 \mathrm{Ac}$ & $0,832 \mathrm{Ab}$ \\
\hline G3 & $0,825 \mathrm{Bb}$ & 0,836 Aa & $0,843 \mathrm{Aab}$ & $0,844 \mathrm{Aa}$ \\
\hline G4 & $0,832 \mathrm{Aab}$ & $0,834 \mathrm{Aa}$ & $0,846 \mathrm{Aa}$ & $0,846 \mathrm{Aa}$ \\
\hline G5 & $0,836 \mathrm{Aa}$ & $0,837 \mathrm{Aa}$ & $0,838 \mathrm{Ab}$ & $0,841 \mathrm{Aa}$ \\
\hline
\end{tabular}

Para cada regime de irrigação, letras maiúsculas comparam níveis de potássio dentro de genótipos $(\mathrm{G})$ e letras minúsculas comparam genótipos dentro de cada nível de potássio. RI1 irrigação diária; RI2 - irrigação suspensa até o aparecimento de sintomas iniciais de deficiência hídrica; $\mathrm{K} 0$ - sem adição de $\mathrm{K}_{2} \mathrm{O}$; $\mathrm{K} 1$ - adição de $200 \mathrm{mg} \cdot \mathrm{dm}^{-3} \mathrm{~K}_{2} \mathrm{O}$.

\section{CONCLUSÕES}

Os genótipos submetidos à suspensão da irrigação apresentaram modificações expressivas em todas as características fisiológicas.

A adição de $\mathrm{K}$ diminui os efeitos da deficiência hídrica nos cinco genótipos testados.

O genótipo G1 é o mais tolerante e o genótipo G2 o mais sensível à deficiência hídrica.

A adubação potássica pode amenizar os efeitos negativos da deficiência hídrica nos estádios iniciais de crescimento dos genótipos testados.

\section{REFERÊNCIAS}

ARQUERO, O.; BARRANCO, D.; BELLONCH, M.

Potassium starvation increases stomatal condutance in olive trees. HortScience, Alexandria, v. 41, n. 2, p. 433-436, 2006.

Cerne, Lavras, v. 19, n. 4, p. 603-611, out./dez. 2013 
BARROS, N. F.; NOVAIS, R. F.; NEVES, J. C. L. Fertilização e correção do solo para o plantio de eucalipto. In: BARROS, N. F.; NOVAIS, R. F. (Ed.). Relação solo-eucalipto. Viçosa, MG: Folha de Viçosa, 1990. p. 127-186.

CATSKY, J. Water saturation déficit: relative water content. In: SLAVIK, B. (Ed.). Methods of studying plant water relations. Berlin: Springer-Verlag, 1974. p. 136-154.

CHAVES, M. M.; MAROCO, J. P.; PEREIRA, J. S. Understanding plant responses to drought: from genes to the whole plant. Functional Plant Biology, Camberra, v. 30, n. 3 , p. 239-264, 2003.

CHAVES, M. M.; PEREIRA, J. S.; MAROCO, J.; RODRIGUES, M. L.; RICARDO, P. P.; OSÓRIO, M. L.; CARVALHO, I.; FARIA, T.; PINHEIRO, C. How plants cope with water stress in the field photosynthesis and growth? Annals of Botany, Oxford, v. 89, n. 7, p. 907-916, 2002.

DITMAROVÁ, L.; KURJAK, D.; PALMROTH, S.; KMEŤ, J.; STŘELCOVÁ, K. Physiological responses of Norway spruce (Picea abies) seedlings to drought stress. Tree Physiology, Oxford, v. 30, n. 2, p. 205-213, 2010.

FANAEI, H. R.; GALAVI, M.; KAFI, M.; BONJAR, G. Amelioration of water stress by potassium fertilizer in two oilseed species. International Journal of Plant Production, Gorgan, v. 3, n. 2, p. 41-54, 2009.

FERREIRA, D. F. Sistema de análises de variância para dados balanceados: SISVAR 4.1. Lavras: UFLA, 2000. Software.

FLEXAS, J.; BOTA, J.; ESCALONA, J. M.; SAMPOL, B.; MEDRANO, H. Effects of drought on photosynthesis in grapevines under field conditions. Functional Plant Biology, Dordrecht, v. 29, n. 4, p. 461-471, 2002.

FLEXAS, J.; MEDRANO, H. Drought-inhibition of photosynthesis in $\mathrm{C}_{3}$ plants: stomatal and non-stomatal limitations revisited. Annals of Botany, Oxford, v. 89, n. 2, p. 183-189, 2002.

JALEEL, C. A.; MANIVANNAN, P.; LAKSHMANAN, G. M. A.; GOMATHINAYAGAM, M.; PANNEERSELVAM, R. Alterations in morphological parameters and photosynthetic pigment responses of Catharanthus roseus under soil water deficits. Colloids and Surfaces B: Biointerfaces, Amsterdam, v. 61, p. 298-303, 2008.

MARSCHNER, H. Mineral nutrition of higher plants. San Diego: Academic, 1995. 888 p.

MERCHANT, A.; CALLISTER, A.; ARNDT, S.; TAUSZ, M.; ADAMS, M. Constrasting physiological responses of six Eucalyptus species to water deficit. Annals of Botany, Oxford, v. 100, n. 7, p. 1507-1515, 2007.

MIELKE, M. S.; OLIVA, M. A.; BARROS, N. F.; PENCHEL, R. M.; MARTINEZ, C. A.; ALMEIDA, A. C. Stomatal control of transpiration in the canopy of clonal Eucalyptus grandis plantation. Trees, Berlin, v. 13, p. 152-160, 1999.

MISSON, L.; LIMOUSIN, J. M.; RODRIGUEZ, R.; LETTS, L. G. Leaf physiological responses to extreme droughts in Mediterranean Quercus ilex Forest. Plant, Cell and Environment, Oxford, v. 33, n. 11, p. 1898-1910, Nov. 2010.

NEVES, J. C. L.; GOMES, J. M.; NOVAIS, R. F. Fertilização mineral de mudas de eucalipto. In: BARROS, N. F.; NOVAIS, R. F. (Ed.). Relação solo-eucalipto. Viçosa, MG: Folha de Viçosa, 1990. p. 99-126.

NGUGI, M. R.; DOLEY, D.; HUNT, M. A.; RYAN, P.; DART, P. Physiological responses to water stress in Eucalyptus cloeziana and E. argophloia seedlings. Trees, Berlin, v. 18, p. 381-389, 2004.

NGUGI, M. R.; HUNT, M. A.; DOLEY, D.; RYAN, P.; DART, P. Dry matter production and allocation in Eucalyptus cloeziana and Eucalyptus argophloia seedlings in response to soil water deficits. New Forests, West Lafayette, v. 26, n. 2, p. 187-200, 2003a.

NGUGI, M. R.; HUNT, M. A.; DOLEY, D.; RYAN, P.; DART, P. Effects of soil water availability on water use efficiency of Eucalyptus cloeziana and Eucalyptus argophloia plants. Australian Journal of Botany, Camberra, v. 51, n. 2, p. 59166, 2003 b.

NOVAIS, R. F.; BARROS, N. F.; NEVES, J. C. L. Nutrição mineral do eucalipto. In: BARROS, N. F.; NOVAIS, R. F. (Ed.). Relação solo-eucalipto. Viçosa, MG: Folha de Viçosa, 1990. p. 25-98.

Cerne, Lavras, v. 19, n. 4, p. 603-611, out./dez. 2013 
PEREIRA, M. R. R.; KLAR, A. E.; SILVA, M. R.; SOUZA, R. A.; FONSECA, N. R. Comportamento fisiológico e morfofisiológico de clones de Eucalyptus urograndis submetidos a diferentes níveis de água no solo. Irriga, Botucatu, v. 11, n. 4, p. 518-531, 2006.

PITA, P.; CAÑAS, I.; SORIA, F.; RUIZ, F.; TOVAL, G. Use of physiological traits in tree breeding for improved yield in drought-prone environments: the case of Eucalyptus globulus. Investigación Agraria, Madrid, v. 14, n. 3, p. 383393, 2005.

RAMPINO, P.; PATALEO, S.; GERARDI, C.; MITA, G.; PERROTTA, C. Drought stress response in wheat: physiological and molecular analysis of resistant and sensitive genotypes. Plant, Cell and Environment, Oxford, v. 29, n. 12, p. 243-252, 2006.

RASCHER, U.; LIEBIG, M.; LÜTTGE, U. Evaluation of instant light-response curves of chlorophyll fluorescence parameters obtained with a portable chlorophyll fluorometer on site in the Field. Plant, Cell and Environment, Oxford, v. 23, n. 12, p. 1397-1405, 2000.

ROLANDO, C. A.; LITTLE, K. M. Measuring water stress in Eucalyptus grandis Hill ex Maiden seedlings planted into pots. South African Journal of Botany, Grahamstown, v. 74, n. 1, p. 13-18, 2008.

ROLANDO, C. A.; LITTLE, K. M. Using chlorophyll fluorescence to determine stress in Eucalyptus grandis seedlings. Southern African Forestry Journal, Gauteng, v. 197, p. 5-12, 2003.

ROMERO, R. R. Respostas fisiológicas de plantas de Eucalyptus grandis à adubação com potássio ou sódio. 2008. 56 f. Dissertação (Mestrado em Fisiologia e Bioquímica de Plantas) - Escola Superior de Agricultura "Luiz de Queiroz”, Piracicaba, 2008.
SCHOLANDER, P. F.; HAMMEL, H. T.; BRADSTRET, E. D.; HEMMINGSEN, E. A. Sap pressure in vascular plants. Science, Cambridge, v. 148, p. 339-346, 1965.

SEGHATOLESLAMI, M. J.; KAFI, M.; MAJIDI, E. Effect of drought stress at different growth stages on yield and water use efficiency of five proso millet (Panicum miliaceum L.) genotypes. Pakistan Journal of Botany, Karachi, v. 40, n. 4, p. 1427-1432, 2008.

SILVA, M. A.; JIFON, J. L.; SILVA, J. A. G. da; SHARMA, V. Use of physiological parameters as fast tools to screen for drought tolerance in sugarcane. Brazilian Journal of Plant Physiology, Londrina, v. 19, n. 3, p. 193-201, 2007.

SILVA, M. R.; KLAR, A. E.; PASSOS, J. R. Efeitos do manejo hídrico e da aplicação de potássio nas características morfofisiológicas de mudas de Eucalyptus grandis (Hill ex. Maiden). Irriga, Botucatu, v. 9, n. 1, p. 31-40, 2004.

TATAGIBA, S. D.; PEZZOPANE, J. E. M.; REIS, E. F. Relações hídricas e trocas gasosas na seleção precoce de clones de eucalipto para ambientes com diferenciada disponibilidade de água no solo. Floresta, Curitiba, v. 38, n. 2, p. $387-400,2008$.

TEIXEIRA, P. C.; GONÇALVES, J. L. M.; ARTHUR JUNIOR, J. C. Crescimento e partição de matéria seca de mudas de eucalipto em função da adubação potássica e água do solo. Revista Ceres, Viçosa, v. 53, n. 510, p. 672-676, 2006.

TEIXEIRA, P. C.; GONÇALVES, J. L. M.; ARTHUR JUNIOR, J. C.; DEZORDI, C. Eucalyptus sp. seedling response to potassium fertilization and soil water. Ciência Florestal, Santa Maria, v. 18, n. 1, p. 47-63, 2008.

TEIXEIRA, P. C.; LEAL, P. G. L.; BARROS, N. F.; NOVAIS, R. F. Nutrición potásica y relaciones em plantas de Eucalyptus spp. Bosque, Valdivia, v. 16, n. 2, p. 61-68, 1995.

Recebido: 3 de março de 2011; aceito: 24 de maio de 2013.

Cerne, Lavras, v. 19, n. 4, p. 603-611, out./dez. 2013 
